Accepted by ApJ Letters April 9, 2009.

\title{
MIXED POPULATIONS IN GLOBULAR CLUSTERS: ET TU, 47 TUC? $^{1}$
}

\author{
J. Anderson ${ }^{2}$, G. Piotto ${ }^{3}$, I. R. King ${ }^{3}$, L. R. Bedin ${ }^{2}$, and P. Guhathakurta ${ }^{4}$
}

\begin{abstract}
We exploit the large number of archival HST images of 47 Tuc to examine its subgiant branch (SGB) and main sequence (MS) for signs of multiple populations. In the cluster core, we find that the cluster's SGB exhibits a clear spread in luminosity, with at least two distinct components: a brighter one with a spread that is real but not bimodal, and a second one about 0.05 mag fainter, containing about $10 \%$ of the stars. In a less crowded field $6^{\prime}$ from the center, we find that the MS is broadened much more than can be accounted for by photometric errors, and that this broadening increases at fainter magnitudes.
\end{abstract}

Subject headings: globular clusters: individual (NGC 104) — Hertzsprung-Russell diagram

\section{Introduction}

For half a century the standard doctrine that each globular cluster (GC) consists of stars born at the same time out of the same material has contributed enormously to our

\footnotetext{
${ }^{2}$ Space Telescope Science Institute, 3800 San Martin Drive, Baltimore, MD 21218; [jayander,bedin]@stsci.edu

${ }^{3}$ Astronomy Dept., Padova Univ., Vic. Osservatorio 2, 35122, PD, Italy; piotto@pd.astro.it

${ }^{4}$ Astronomy Dept., Univ. of Washington, Box 351580, Seattle, WA 98195-1580, USA; king@astro.washington.edu

${ }^{5}$ UCO/Lick Observatory, University of California Santa Cruz, 1156 High St., Santa Cruz, CA 95064; raja@ucolick.org

${ }^{1}$ Based on observations with the NASA/ESA Hubble Space Telescope, obtained at the Space Telescope Science Institute, which is operated by AURA, Inc., under NASA contract NAS 5-26555.
} 
understanding of stellar evolution. In recent years, however, discoveries made largely by our group have contradicted this paradigm, and the study of GC populations has moved in a new direction. It had already been noted that the CMDs of M54 (Sarajedini \& Layden 1995) and $\omega$ Cen (Lee et al. 1999 and Pancino et al. 2000) showed evidence of multiple redgiant branches, but the new era began with the discovery (Bedin et al. 2004) that the main sequence of $\omega$ Centauri is split into two branches. Spectroscopy showed (Piotto et al. 2005) that the metallicities made sense only if the bluer branch had a strong helium enhancement.

Next, discoveries based on photometry of unparalleled accuracy, on HST/ACS images, have shown that NGC 2808 has three parallel main sequences (MSs) (Piotto et al. 2007), and NGC 1851 has a split subgiant branch (Milone et al. 2008a). More recently, we have found split sub-giant branches (SGBs) in NGC 6388, NGC 6656, and other Galactic GCs (see Piotto 2009 for an update), and in many clusters of the Large Magellanic Cloud (Milone et al. 2008b).

We note in passing that Galactic GCs have also shown abundance peculiarities (see Gratton et al. 2004 for the most recent review) that also suggest multiplicities of population, but here we will be discussing only photometric peculiarities.

One striking characteristic common to all the clusters that we have mentioned is that they are among the most luminous GCs in the Galaxy. The most massive cluster that has not yet been investigated with high-precision photometry is 47 Tucanae. Fortunately, 47 Tuc has been used for HST calibration, and a wealth of archival data is available. In this paper we will examine two fields in 47 Tuc. In the central field, where the star numbers are greatest, we will examine the subgiant branch, and in an outer field, where the crowding of faint stars is minimal, we will take a close look at the stars of the main sequence.

\section{Multiple SGBs in the Cluster core}

The core of 47 Tuc has been observed several times with HST's Advanced Camera for Surveys. The archive contains a very large set of observations taken with the HRC, but there are too few stars in the small HRC field to get good statistics on the SGB population, so we focus here on the WFC data. Five data sets were used here (program-ID, filters, number of exposure, exposure time): (1) GO-9028 F475W 20×60 s, (2) GO-9443 F475W 5×60 s, (3) GO-9503 F475W $1 \times 60 \mathrm{~s}$, (4) GO-9281 F435W $9 \times 105 \mathrm{~s}$ and F625W 20×65 s, (5) GO-10775 F606W $4 \times 50 \mathrm{~s}$ and F814W $5 \times 50 \mathrm{~s}$.

The _flt images from the $H S T$ pipeline were reduced using the program described in Anderson \& King (2006, AK06), which uses for each filter a spatially varying array of PSFs, 
plus a "perturbation PSF" tailored to each exposure to compensate for the effect of focal variations. The photometry was put into the ACS/WFC Vega-mag system according to the procedure given by Bedin et al. (2005), and using the encircled energy and zero points given by Sirianni et al. (2005).

Features on the subgiant branch are usually difficult to discern, since the fiducial sequence moves rapidly in both color and magnitude. Here, we can take advantage of the fact that for the metallicity and age of 47 Tuc, the SGB is nearly horizontal in F475W, so that all SGB stars should have the same F475W flux, within observational errors.

The first three programs that we listed give us a F475W observation for each star. The next two programs each give us a useful color measurement. We thus have for each star three independent F475W magnitude measurements and two independent measurements of the color, $\left(m_{\mathrm{F} 435 \mathrm{~W}}-m_{\mathrm{F} 625 \mathrm{~W}}\right)$ and $\left(m_{\mathrm{F} 606 \mathrm{~W}}-m_{\mathrm{F} 814 \mathrm{~W}}\right)$. To ensure the best possible photometry, when we reduced each of the images we retained only the unsaturated stars that gave a good fit to the PSF (the QSEL flag, see AK06). This did a good job of preventing crowded stars with compromised photometry from entering our lists (particularly important in the crowded core of 47 Tuc). Next, we made a master list of stars from the GO-9028 data set, and cross-identified the photometry in all the other data sets with this.

We now had seven independent fluxes for each star: three in F475W and one in each of F435W, F625W, F606W, and F814W. Figure 1 shows the six CMDs that can be constructed from the three independent $m_{\mathrm{F} 475 \mathrm{~W}}$ magnitudes and the two colors. In the top left plot, which shows the best photometry, we arbitrarily select three groups of stars: those well below the SGB (red open triangles), those in the lower half of the main SGB (green open circles), and those in its upper half (blue filled circles). The black lines in the first CMD delineate the regions chosen. We plot these same stars in all the other diagrams. In the first column, the stars are plotted according to their observed colors in the GO-9281 data set; in the second column we use the colors from GO-10775. The three panels down each column use the F475W magnitude from a different one of the three independent F475W data sets, which were taken at different pointings, times, and orientations. The fact that the red-triangle stars lie below the main SGB in all the plots means that they truly are fainter. Similarly, the fact that the blue filled-circle stars are brighter than the green open-circle ones also confirms that the magnitude difference is significant and shows an intrinsic vertical spread in the main SGB.

The histograms on the right show the distribution of the stars in F475W magnitude as measured in the three data sets. It is clear that the stars that were shown as blue filled circles are indeed systematically brighter than those that were shown as green open circles. There is no apparent bimodality in these two groups, but our multiple independent observations 
demonstrate that the observed spread is not consistent with random photometric errors. The SGB stars that were shown as red triangles are considerably fainter ( $\sim 0.05$ magnitude) than the other two groups; they make up roughly $10 \%$ of the SGB population.

47 Tuc has also been extensively observed in an outer field, located $6^{\prime}$ West of the cluster center. These observations will be used in the following section to study the MS. We also examined the SGB there, using the short exposures: the SGB stars are not numerous enough for a detailed study, but they show strong indications of a vertical spread similar to what we saw in the inner field. Studying the radial gradient of the SGB populations will require a larger survey than our single outlying ACS field can provide.

\section{Intrinsic Breadth of the MS}

While the density in the outer field at $6^{\prime}$ is too low for studying the SGB in detail, it is ideal for examining the structure of the main sequence. In addition to the short exposures mentioned briefly above, there are also more than a hundred deep exposures, taken at a wide variety of epochs, offsets, and orientations, for both calibration and scientific purposes. In toto, there are 115 images in F606W, with exposure times from 339 to $1400 \mathrm{~s}$, from 10 different GO numbers, and 32 in F814W, of 339 to 1500 s, from seven different GO's.

We will use this material to show that the MS of 47 Tuc has an intrinsic width much greater than the spread that would be produced by observational error alone; the key to this is of course establishing as reliably as possible how small our observational errors are. With data sets coming from such varied circumstances, combining them to get an accurate color and magnitude for each star is a complicated procedure, and it would be hopelessly naive to use any sort of $\sqrt{N}$ rule; what we do instead is to divide our data into two halves, each having random orientations and offsets, and estimate our final errors from the star-by-star agreement between the results from the two halves.

Our first step, however, was to remove the one systematic error that we could correct, the small photometric error that depends on position on the detector chips. Using the entire outer-field data set, we derived a residual for each star in each image, by comparing each measured F606W magnitude with the average F606W magnitude of that star in all 115 F606W images, and similarly for each F814W image against the average from all $32 \mathrm{~F} 814 \mathrm{~W}$ images. We then examined the residuals for each filter as a function of location on each chip, and constructed for each filter a $128 \times 128$ array of corrections (spaced at 32-pixel intervals), which we applied to our photometry. These corrections were typically 0.005 mag in size; they compensate for the fact that our $9 \times 10$ array of library PSFs does not allow perfectly 

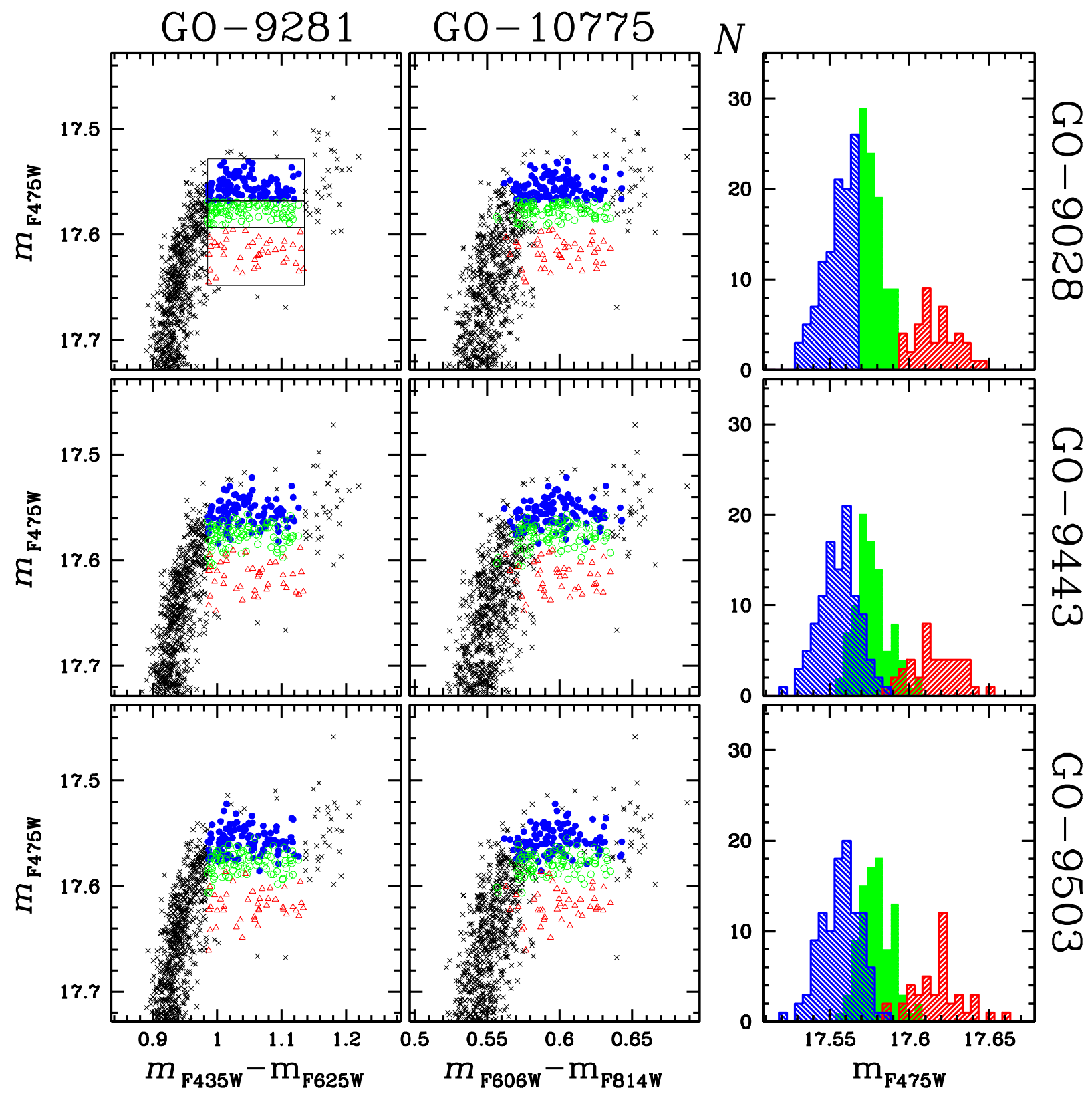

Fig. 1. - Independent observations demonstrate that the intrinsic broadening of the SGB of 47 Tuc must be real (see text). 
for all PSF variations.

We made the division of our data set into halves as follows. We first divided the images into four sets: the first half of the F606W images (V1) and the second half (V2), and the first and second halves of the F814W images (I1 and I2). We constructed a CMD from the V1 and I1 data, and another independent one from the V2 and I2 data. In each CMD we drew in the same main-sequence ridge line (MSRL), and subtracted from the color of each star the color of the MSRL at its measured V magnitude, thus creating for each half of the data a vertical sequence with a spread in color.

Figure 2 provides some orientation for our star measurements in the outer field. Panel (a) shows a CMD of 47 Tuc, with the faint stars coming from the analyzed outer field, and the upper part coming from the central field (where there are more bright stars). Panel (b) is a blowup identifying the magnitude range with which the analysis in this section deals. Stars in magenta are non-members, according to their proper motions; they are not used in what follows. (Most of the non-members are SMC stars in the background. The cluster members at lower left are white dwarfs.) The dashed red line in panel (b) is the locus of equal-mass MS binaries.

Figure 3 shows the results of our analysis of the deep outer-field data. Panel (a) shows the color residual, $\Delta_{1}$, for each the star as obtained from the first half of the data, in the sense $\left(m_{F 606 W}-m_{F 814 W}\right)_{o b s}-\left(m_{F 606 W}-m_{F 814 W}\right)_{M S R L}$. The second color residual $\Delta_{2}$, in panel (b), shows the same quantity for the second half of the data. These two estimates of the color residual of each star relative to the MSRL should be entirely independent of each other. The vertical black lines in panel (a) are drawn in such a way that there are equal numbers of points to the blue, to the red, and between the two lines, and the points on either side are color-coded accordingly. In panel (b) each star retains the color-coding that was assigned to it in panel (a); it is striking how well the ordering of these colors is maintained in this independent sample.

To emphasize the consistency of the results from the two halves of our data, in panel (c) we plot $\Delta_{1}$ against $\Delta_{2}$. The tight correlation shows that each star is measured to be bluer or redder than the MSRL by nearly the same amount in the independent halves of the data. This is one way of demonstrating the reality of the apparent color width of the MS. The spread from lower left to upper right is the color spread of the individual stars, while the spread in the perpendicular direction is indicative of our measuring error. (It is interesting also to note, in the upper two sections, the unilateral spread of binaries redward of the MS; this fails to be evident in the lower two sections only because of the increased spread of the MS stars.) 


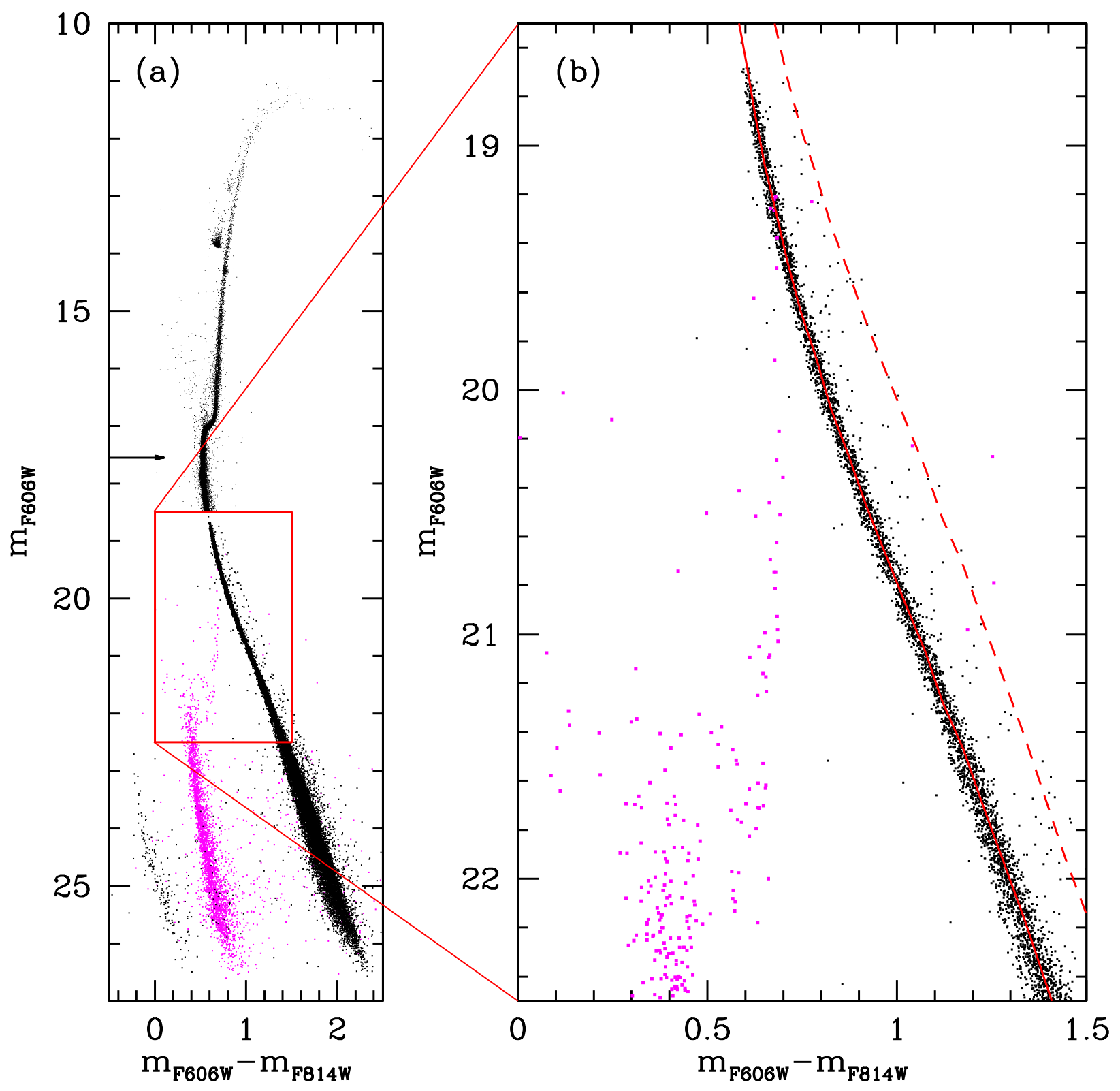

Fig. 2.- (a) The full CMD, with proper-motion non-members in magenta. We mark the location of the main-sequence turnoff with an arrow just below $m_{\mathrm{F} 606 \mathrm{~W}} \sim 17.5$. (b) Region with high-quality deep photometry, with the MSRL and equal-mass-binary line drawn in. 

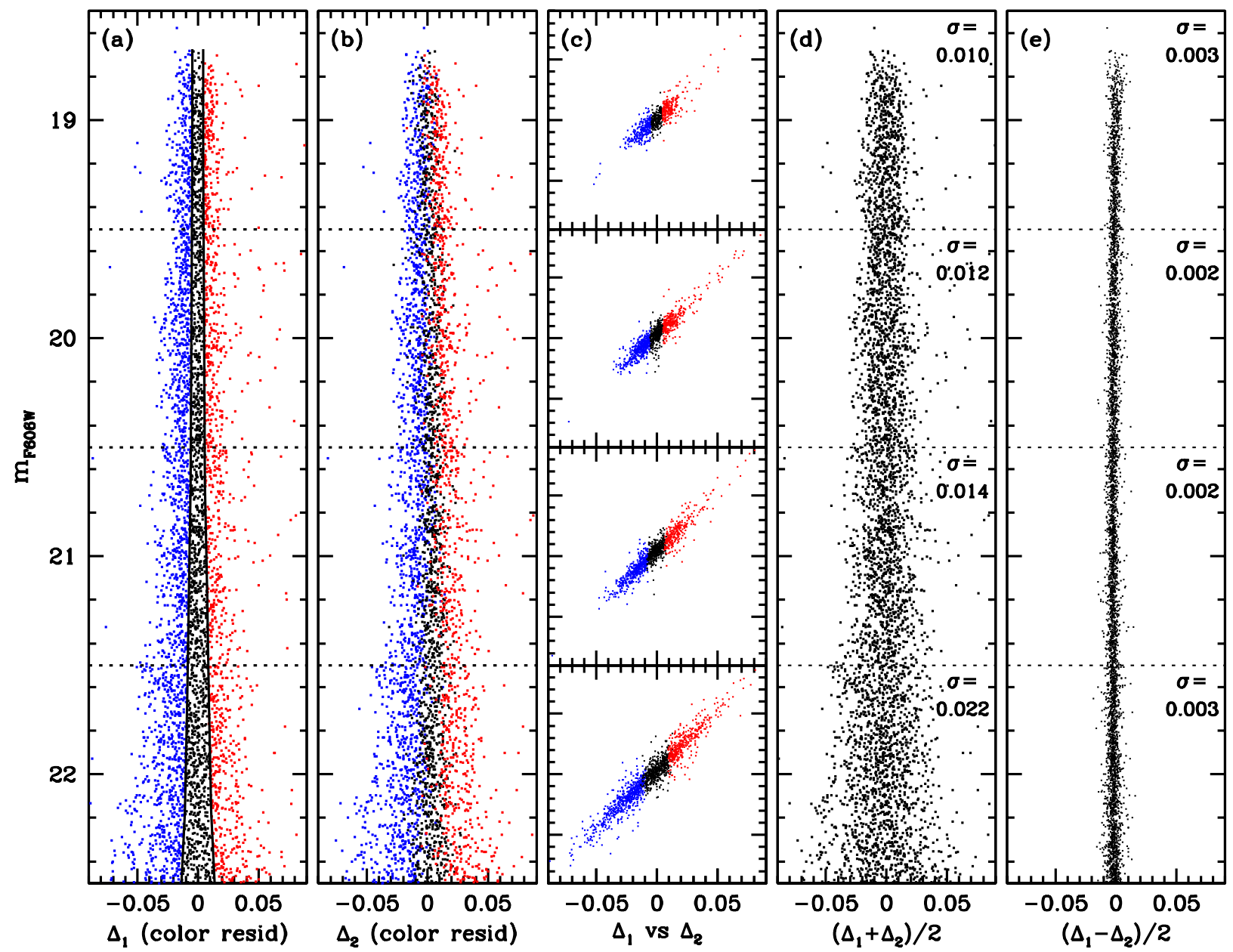

Fig. 3.- (a) Straightened sequence for the 1st half of the data, divided into blue, middle, and red thirds. (b) Straightened sequence for the 2nd half of the data; the color for each star as was assigned in the previous panel. (c) Correlation of the two independent color residuals, for the four magnitude bins. (d) Mean values of color residuals. (e) Estimates of color errors. 
The next two panels show the same relationships in a more quantitative way. The quantities $\Delta_{1}$ and $\Delta_{2}$ are independent measurements of the color offset between each star and the MSRL. To obtain the best estimate of the $\Delta$ for each star we average the two estimates. To estimate the error in this average, we assume that $\Delta_{1}$ and $\Delta_{2}$ are Gaussian

deviates with rms $\sigma$ and that the difference between them should be $\sim \sqrt{2} \sigma$, and that the error in the average should then be $\sigma / \sqrt{2}$, or $\sim\left|\Delta_{1}-\Delta_{2}\right| / 2$. In panels (d) and (e), each of the quoted sigmas was derived by taking half the the distance between the 16th and 84th percentiles of the distribution in that magnitude bin.

The error estimates are admittedly that; in the presence of small systematic errors of $\sim 0.005$ magnitude in each exposure due to uncompensated-for breathing and other effects, it is difficult to be sure of the exact size of our errors. Another more tediously derived estimate (based on a study of many different pairings of F606W and F814W exposures) had, for example, an increase of the error sigma from 0.0012 to 0.0028 from brightest stars to faintest. But what is unmistakably true in any case is that the observed spread in MS colors is 5 to 10 times what can be explained by measuring error. The main sequence of 47 Tuc has a considerable intrinsic breadth. Furthermore, the increase in breadth from bright to faint is greater than can be explained by an increase in measurement error, and must therefore be real.

Finally, we note that the average MSRL residuals in panel (d) show no structure; like the upper branch of the split SGB, the MS shows no signs of a split.

\section{Discussion}

The CMDs of 47Tuc presented in this paper show that this cluster hosts a complex stellar population, as do all the other massive $\left(M>10^{6} m_{\odot}\right)$ clusters investigated so far. In particular: i) The SGB is split into two SGBs, with the upper SGB showing in addition a clear vertical broadening. ii) The MS also shows an intrinsic broadening, which tends to increase as we go from 3 to 6 magnitudes below the turn-off.

The complex SGB morphology is hard to interpret without a detailed chemical analysis of the stars in the different SGBs. Very likely it is the result of a combination of metallicity, age, and possibly He variations, as in the case of $\omega$ Cen (Villanova et al. 2007) and NGC 1851 (Cassisi et al. 2008).

A broadening of the MS at $>3$ magnitudes below the turn-off could in principle be due to some combination of 1) a depth effect, 2) binaries, 3) differential reddening, 4) a spread in metallicity, or 5) a spread in He. A simple King model shows that the line-of- 
sight distribution of stars at this location in the cluster would imply a spread of less than 0.005 magnitude in the photometry, and a spread of 0.001 in color relative to the MSRL. This clearly cannot explain the spread that we see. As for a binary explanation, equalmass binaries would have $\Delta \gtrsim 0.15$ mag in color, so the small symmetric spread observed would imply a conspiracy of a very high binary fraction and a distribution with very low secondary/primary mass ratios. Besides, it seems even more unlikely that any distribution of binaries could explain the symmetry of the color spread. Variable reddening is also unlikely to explain the observed spread, since the total reddening is only $E(B-V)=0.04$ (Harris 1996).

We have used isochrones from the Teramo group (Pietrinferni et al. 2004), recalculated by S. Cassisi for different helium contents, to estimate the $\mathrm{Fe}$ and He dispersion that could lead to the observed color spread in the MS. For $m_{\text {F606W }}$ between 20 and 21 , we have an average $\left(m_{\mathrm{F} 606 \mathrm{~W}}-m_{\mathrm{F} 814 \mathrm{~W}}\right)$ color dispersion of $\sim 0.013$ magnitude. If the observed dispersion is due solely to a dispersion in iron (with standard He content), then it would imply $\Delta[\mathrm{Fe} / \mathrm{H}]$ $\simeq 0.1$. If He is the sole cause of the MS broadening, then a He dispersion $\Delta Y \sim 0.026$ would be implied.

It should come as no surprise that a cluster main sequence has an intrinsic spread in color. Indeed, there had to be some limit to the homogeneity in the cloud that formed it, even under the single-population paradigm. It is still unclear, though, whether the spread that we see in 47 Tuc is primordial or whether it is indicative of a more complex formation history. It is important to emphasize that we do not have such a high-precision data set for any other cluster, so it is simply not possible to search other clusters for MS broadening at the level of accuracy needed to find a color spread of $\sim 0.01$. Nevertheless, the SGB spread of $>0.05$ magnitude in $\mathrm{F} 475 \mathrm{~W}$ is impossible to explain in terms of a simple, single stellar population.

Looking ahead, a spectroscopic study of the stars in the different SGB populations could resolve whether the vertical spread is due to age or metallicity (or both). Also, by surveying how the fraction of stars in the SGB populations varies over a larger radial region, we could explore whether the multiple populations are better explained by a merger or self-enrichment scenario. Such a survey would have to cover much more than a single outer ACS field, and would require proper-motion cleaning to distinguish the minority SGB populations from field and SMC stars.

J.A. and I.R.K. acknowledge support from STScI grants GO-9444 and GO-10101. GP acknowledges support by PRIN2007 and ASI under the program ASI-INAF I/016/07/0. 


\section{REFERENCES}

Anderson, J., \& King, I. R. 2006, ACS ISR 2006-01

Bedin, L. R., Piotto, G., Anderson, J., Cassisi, S., King, I. R., Momany, Y., \& Carraro, G. 2004, ApJL, 605, L125

Bedin, L. R., Cassisi, S., Castelli, F., Piotto, G., Anderson, J., Salaris, M., Momany, Y., \& Pietrinferni, A. 2005, MNRAS, 357, 1038

Cassisi, S., Salaris, M., Pietrinferni, A., Piotto, G., Milone, A. P., Bedin, L. R., \& Anderson, J. 2008, ApJL, 672, L115

Gratton, R., Sneden, C., \& Carretta, E. 2004, ARA\&A, 42, 385

Harris, W. E. 1996, AJ, 112, 1487 (February 2003 update).

Lee, Y.-W., Joo, J.-M., Sohn, Y.-J., Rey, S.-C., Lee, H.-C., \& Walker, A. R. 1999, Nature, 402,55

Milone, A. P., et al. 2008a, ApJ, 673, 241

Milone, A. P., Bedin, L. R., Piotto, G., \& Anderson, J. 2008b, A\&A, in press (arXiv:0810.2558)

Pancino, E., Ferraro, F. R., Bellazzini, M., Piotto, G., \& Zoccali, M. 2000, ApJ, 534, L83

Pietrinferni, A., Cassisi, S., Salaris, M., \& Castelli, F., 2004, ApJ, 612, 168

Piotto, G., et al. 2005, ApJ, 621, 777

Piotto, G., Bedin, L. R., Anderson, J., King, I. R., Cassisi, S., Milone, A. P., Villanova, S., Pietrinferni, A., \& Renzini, A. 2007, ApJ, 661, L53

Piotto, G. 2009, IAU Symposium No. 258, in press (astro-ph, arXiv:0902.1422)

Sarajedini, A., \& Layden, A. C. 1995, AJ, 109, 1086

Sirianni, M., et al. 2005, PASP, 117, 1049

Villanova, S., et al. 2007, ApJ, 663, 296 